\title{
Routine betamethasone therapy of non-hydropic fetuses with CPAM - the way to improve perinatal outcome?
}

\author{
Kunochova I, Krizko M, Papcun P, Gabor M, Alfoldi M, Feriancova M, Ferianec V \\ 2nd Department of Obstetrics and Gynecology, University Hospital Bratislava, Bratislava, Slovakia. \\ ferianec@gmail.com
}

\begin{abstract}
Authors discuss novel approach to the management of fetal congenital pulmonary cystic malformation (CPAM) and possible benefit of routine administration of betamethasone, which is currently recommended only for severe cases. The article presents authors' own experience with antenatally diagnosed CPAM and describes 4 cases of prenatally diagnosed CPAM without hydrops treated by two doses of betamethasone at 21-31 weeks of gestation with the aim of improving the perinatal prognosis by effect on not only mortality but also postnatal morbidity. Article also summarizes current knowledge on all aspects of the prenatal CPAM focusing on its treatment options. Data were obtained from the literature search based on the PubMed and Scopus database with additional search of particular articles from reference list of the selected publications. All 4 patients from the case reports showed regression of the pulmonary mass after maternal administration of betamethasone with $100 \%$ survival rate. Even though it is not possible to say if the CPAM regression was due to the betamethasone treatment, we did not observe any complication in relation to the treatment and all cases survived until discharge. During the literature search, we did not find any data on betamethasone administration in non-hydropic fetuses with CPAM in relation to the overall perinatal and postnatal morbidity, neither data comparing the outcome between the treated versus observed only fetuses.

Routine betamethasone treatment should be discussed in antenatally diagnosed CPAM cases without fetal hydrops in order to reduce the perinatal morbidity associated with CPAM (Tab. 1, Ref. 47). Text in PDF www.elis.sk.

KEY WORDS: betamethasone, CPAM (congenital pulmonary adenomatoid malformation), fetal therapy.
\end{abstract}

\section{Introduction}

Congenital pulmonary cystic malformation (CPAM), previously known as cystic adenomatoid malformation (CCAM), refers to benign multicystic mass of nonfunctioning pulmonary tissue that is usually restricted to one lobe of the lung (1). It represents an abnormality in lung development characterized by the lack of normal alveoli and abnormal proliferation of terminal respiratory bronchioles (2). The exact etiology remains unknown. Histopathology confirms bronchial atresia with absence of normal alveoli due to decreased apoptosis during the embryological development between 7 and 15 weeks of gestation (3). CPAM is usually isolated and sporadic, rarely it can be associated with other anomalies, such as cardiac anomalies, renal agenesis or dysgenesis, gastrointestinal atresia or skeletal anomalies associated particularly with the microcystic form of CPAM (4). Prognosis depends on the CPAM size rather than lesion type. However, natural history of the lesion can differ, as some may come to spontaneous regression, others

2nd Department of Obstetrics and Gynecology, University Hospital Bratislava, Ružinovská 6, 82606 Bratislava, Slovakia

Address for correspondence: V. Ferianec, MD, Assoc Prof, PhD, 2nd Department of Obstetrics and Gynecology, University Hospital Bratislava, Ruzinovska 6, SK-826 05 Bratislava, Slovakia.

Phone: +4212 905385670 stagnate or progress to grow (5). Generally, the CPAM is usually highly unpredictable in its growth potential between 18 and 26 weeks of gestation (gw). The fastest growth appears between 20 and 25 weeks with a peak of CVR (CPAM volume ratio) occurring at $25 \mathrm{gw}$ (6). There is a plateau in CPAM growth beginning at 25 gw with a decrease in the CVR after 25 weeks gestation reflecting continued fetal growth (7).

CPAM is responsible for 25-30\% of congenital lung malformations and is the most common prenatally diagnosed cystic lung disease (8). Incidence ranges from 1:11 000 to 1:35 000 pregnancies and live births, male fetuses are more likely to be affected (9). The reported perinatal mortality of antenatally diagnosed CPAM has decreasing trend over the world, ranging from $49 \%$ in 1992 to $9 \%$ in $2001(8,10)$.

Mortality results mainly from the pulmonary hypoplasia (11). Postnatally is CPAM associated with moderate respiratory symptoms during childhood, persistence of the lesion can cause recurrent infections and even its evolution into malignant processes cannot be excluded $(12,13)$.

The original classification of CPAM according to Stocker et al (14), dividing CPAM lesions into three groups, has been recently extended to five types ( 0 -IV) based on the histopathological image and localization of the malformations at various levels of the airway and lung (15-17). Type 0 CPAM is characterized by bronchial-type airways separated only by abundant mesenchymal 


\section{7-662}

tissue. Types 1 (the bronchial/bronchiolar type) and 2 CPAM (the bronchiolar type) are characterized by cysts $>2 \mathrm{~cm}$ in diameter and multiple small cysts, respectively. In type 3 CPAM (the bronchiolar/alveolar type) the lesion is solid, and not cystic, while type 4 CPAM (the peripheral type) is characterized by peripheral thinwalled, often multiloculated cysts $(15,18)$. Since the classification is based on the histopathology, other classification of Adzick (19) has become widespread in clinical practice as it is based on ultrasound image and gross anatomy. Adzick et al (19) divides CPAM into two major categories according to the predominant component of the lesion. The macrocystic form of CPAM includes cyst(s) $>5$ $\mathrm{mm}$ in diameter while the microcystic form consists of pulmonary mass, which is echogenic and more solid with cysts $<5 \mathrm{~mm}$ in diameter (19). The volume of fetal CPAM lesion is measured by sonography by assessing the pulmonary mass in three dimensions and using the formula length*height*width*0.52 (20).

Despite numerous case reports on CPAM, there is still lack of evidence on reliable antenatal as well as postnatal management of CPAM mainly because of unknown nature and unpredictable prognosis of this pathology.

Prenatal management includes expectancy with observation, minimally invasive procedures such as single thoracocentesis of the cyst or thoracoamniotic shunt in macrocystic lesions. Large microcystic forms may be treated by invasive open fetal surgery or by non-invasive maternal administration of steroids (21). The exact mechanism of steroid therapy is unknown, it is supposed that it accelerates the lung maturation, which is, in case of CPAM, defected during early intrauterine period.

Even though there is no consensus on CPAM treatment, it is mostly recommended to treat patients at high risk of perinatal death defined by the risk factors such as fetal hydrops and value of CVR. The corticosteroid treatment of asymptomatic CPAM is despite the non-invasiveness highly questionable. It is currently not supported considering the relatively good prognosis of CPAM, lack of experience with treatment of these cases (including lack of case reports in the literature) and concerns about the different response to corticosteroid administration. Therefore, we present four cases of prenatally diagnosed non-hydropic fetal CPAM of various severity. With respect to the risk of perinatal morbidity and patients' request for active treatment, all patients have been administered corticosteroids as soon as the diagnosis of CPAM was set. The authors also present current knowledge and literature review on the management of antenatally diagnosed CPAM and discuss different approaches to the antenatal management - routine maternal administration of corticosteroids in order to minimalize the damage of the lung tissue, size of the lung lesions in postnatal period and to prevent the potential evolution of fetal hydrops with possible brain damage.

\section{Patient 1}

32-year-old patient was referred to our center for left intrathoracic echogenic mass apically $(20 \times 11 \times 22 \mathrm{~mm})$ diagnosed by ultrasound at 20 weeks of gestation (gw). Repeated sonography confirmed fetal intrathoracic mass with a mediastinal shift to the right, type 2 CPAM (microcystic form) was diagnosed. The CPAM volume ratio (CVR) at the time of initial diagnosis was 1.5. Ultrasound did not confirm any other congenital anomalies. After a thorough informed consent was obtained, two doses of maternal Betamethasone $12 \mathrm{mg}$ were administrated intramuscularly 24 hours apart at $22 \mathrm{gw}$. Subsequent serial ultrasound examinations were performed to observe the following course of pregnancy with onward regression of CPAM. At $37 \mathrm{gw}$ male fetus was born by caesarean delivery due to two previous caesarean sections, birth weight 2750g, Apgar score 10/10. Newborn was hospitalized at the neonatal intensive care unit for mild dyspnoe and mild desaturation, required distensive respiration without support of oxygen therapy. CT scan of a newborn' chest showed pulmonary lesion $47 \times 17 \times 30 \mathrm{~mm}$ in size. In the 7th month of life, the child underwent thoracoscopic resection of the affected lobe of the lung, histology confirmed the diagnosis of CPAM type II according to the Stoecker's classification.

\section{Patient 2}

36-year-old patient was referred to our tertiary center at $25 \mathrm{gw}$ for a finding of left basal intrathoracic mass. Diagnosis of CPAM was verified by ultrasound and subsequently by MRI. Sonography demonstrated no mediastinal shift, no pericardial effusion, ascites nor polyhydramnios. Other fetal anomalies were not detected by ultrasound. The diagnosis of CPAM mixed type has been set based on the performed examinations. The initial measurements of the mass were $34 \times 23 \times 30 \mathrm{~mm}$. The CVR at that time was 0.6 . After thorough consultation of the case, patient agreed with expectation policy. However, subsequent serial ultrasound examination showed enlarging of the mass, the CVR at $30 \mathrm{gw}$ was 0.85 , measurements $47 \times 28 \times 30 \mathrm{~mm}$, fetus without hydrops or mediastinal shift. With respect to the growth of the mass and potential complications, we agreed on the betamethasone administration at $31 \mathrm{gw}$. During subsequent serial examinations, the mass showed slow regression and CVR decreased to 0.57 at $36 \mathrm{gw}$. At $37 \mathrm{gw}$ labor was induced after premature spontaneous rupture of membranes and male infant was delivered vaginally. Neonate birth weight was $2800 \mathrm{~g}$, Apgar score $10 / 10$. Next course during the hospitalization was without any complications. In the third month of life, a contrast chest CT revealed a residual pulmonary lesion and the patient has recently undergone thoracoscopic surgical treatment.

\section{Patient 3}

30-year-old patient was referred to our center for a finding of intrathoracic mass which involved cystoid lesions in right hemithorax at $26 \mathrm{gw}$. The CVR at referral was 0.24 . MRI image pointed to mixed form of CCAM, no other malformations of the fetus were found. Two doses of betamethasone $12 \mathrm{mg}$ was administrated 24 hours apart at $26 \mathrm{gw}$. The CVR was 0.20 at $30 \mathrm{gw}$ and stagnation of the growth of the mass was observed. The patient was observed without any other treatment. Pregnancy was terminated at $38 \mathrm{gw}$ by elective caesarean delivery due to placenta encroaching. Male infant with a birth weight of $2240 \mathrm{~g}$ and Apgar score 10/10 was 
born. During hospitalization there were no signs of RDS or other respiratory symptoms. In the 6th week of life, the contrast CT of the chest was performed and revealed a pulmonary lesion 29x26x18.5 $\mathrm{mm}$. In the 15 th month of life, the child underwent thoracoscopic resection of the affected lobe.

\section{Patient 4}

28-year-old primigravida at 21 gw was diagnosed with microcystic form of CPAM of the fetus. On ultrasound a hyperechogenic pulmonary mass $22 \times 26 \times 31 \mathrm{~mm}$ was described in the left hemithorax, CVR 0.53. Beside the mass, ultrasound examination revealed also mediastinal shift, however there were no signs of fetal hydrops neither cardiac decompensation. Patient's history did not include any remarkable data. Patient agreed with betamethasone treatment, 2 doses of $12 \mathrm{mg} 24$ hours apart were administrated at $21 \mathrm{gw}$. Subsequent ultrasound at $25 \mathrm{gw}$ showed regression in size $-20 \times 20 \times 23 \mathrm{~mm}, \mathrm{CVR}$ was 0.27 .

At $39 \mathrm{gw}$ labor was induced and female infant was vaginally delivered, birth weight 3230 g, Apgar score 9/10. During the postnatal adaptation neonate did not require any breathing support. In 3rd month of life, contrast CT was performed and CPAM type III according to Stoecker was diagnosed. The patient is currently being prepared for thoracoscopic treatment.

\section{Discussion}

CPAM has become an important study subject due to its different prognosis, clinical manifestation and no clear management guidelines despite the increasing number of prenatally diagnosed cases. The prognosis includes as good course as spontaneous intrauterine resolution of the mass without clinical abnormalities as well as lethal pulmonary hypoplasia, moderate respiratory symptoms postnatally or even progression of the persistent lesion to malignant process $(12,22)$. An extensive review of childhood lung neoplasms revealed that $8.6 \%$ of malignancies were associated with previously documented cystic malformations $(12,22)$. Even though prenatal ultrasound screening and postnatal CT scan represent currently gold standard diagnostic tests, repeated ultrasound examination and fetal magnetic resonance imaging (MRI) allow better and more accurate diagnosis (23).

Differential diagnosis of a fetal lung mass is represented by CPAM, bronchopulmonary sequestration (BPS), congenital high airway obstruction syndrome (CHAOS), congenital lobe emphysema (CLE), congenital diaphragmatic hernia (CDH) and mediastinal tumors (16). A similar picture as a microcystic CPAM may have BPS, which appears in the ultrasonographic image as homogeneous echogenic mass. Doppler's investigation can reveal systemic supply of the mass from the thoracic or abdominal part of the aorta, which is typical of BPS (17). Recent studies show that the distinction between these two entities is not so clear with the discovery of an increasing number of "hybrid" lesions (10).

Generally, the outcome of the fetus with CPAM depends on the complications related to the lesion itself. Lethal pulmonary hypoplasia comes from the compression of the effected ipsilateral lung, polyhydramnios, that may lead to preterm labor, comes from the compression of the esophagus by CPAM mass with inability to swallow, hydrops is related to deterioration of the cardiac venous return due to mediastinal shift (13). Moreover, anemia and cardiac failure with hypotension, which often occur in hydrops, may lead to brain perfusion failure and hypoxic-ischemic changes in fetal brain (24).

Therapeutic options include expectancy with observation or, in case complications occur, invasive techniques such as thoracocentesis, pleuro-amniotic shunt placement, percutaneous ultrasound-guided sclerotherapy, or radiofrequency/laser ablation, for microcystic forms of invasive open fetal surgery or noninvasive maternal administration of steroids, none of which are evidence based (17, 25-30).

According to the current literature data, management decision should be based on some of the defined risk factors and active management should be reserved for the mortality high-risk subgroups. The strongest independent prognostic factor which is considered indication for CPAM treatment has been defined to be hydrops (31). A survival rate of more than $95 \%$ of CPAM cases without hydrops has been reported, whereas death occurred before or after birth in $95 \%$ of CPAM cases with hydrops managed expectantly (32). Crombleholme et al (6) introduced a useful sonographic parameter for assessment of the hydrops - the CPAM volume ratio (CVR), which is CPAM mass volume divided by fetal head circumference. CVR then calculates the ratio of the CPAM volume and fetal head circumference $(($ length $(\mathrm{cm}) \mathrm{x}$ height $(\mathrm{cm}) \mathrm{x}$ width $(\mathrm{cm})$ $x$ 0.52) / head circumference (cm)) (7). CPAM lesions with CVR greater than 1.6 were shown to be at high-risk for fetal hydrops and thus at higher risk for fetal or neonatal death, depending on the gestational age at onset of the hydrops (7). Microcystic form is supposed to be associated with poorer outcome than macrocystic type (19). According to the recent studies, microcystic lesions respond to the administration of maternal betamethasone, resulting in maturation of the fetal lung with good effect on large and growing lesions (33). Steroids decrease the production of lung fluid and increase its reabsorption within the thoracis malformation, thus mimicking the physiological third trimester changes (34). They are indicated for microcystic lesions, while it is unclear whether macrocytic forms respond to this treatment $(35,36)$.

David et al (37) presents an algorithm for prenatal CPAM treatment where he recommends administration of maternal steroid in fetuses less than $32 \mathrm{gw}$ with CVR $>1.6$ or fetuses with hydrops regardless of CVR. Even though the predictive value of CVR in terms of the development of fetal hydrops is generally accepted, no threshold was set for the prediction of fetal/perinatal outcomes. Recent systematic review of the studies reporting on the predictive utility of the CVR suggests that a threshold much lower than $1.6 \mathrm{~cm} 2$ is likely to be of greater utility for outcomes of perinatal relevance. For neonatal outcomes a CVR on the initial ultrasound scan ranging from 0.5 to $1.0 \mathrm{~cm} 2$ appears to have the greatest predictive value (38). Up to now several cases of the microcystic CPAM lesions treated only conservatively with betamethasone have been reported, all of them had hydrops and/ or CVR > 1.6. Tsao et al (2) first published the experience with 
Tab. 1. Review of CPAM cases treated with single course of maternal betamethasone.

\begin{tabular}{|c|c|c|c|c|c|}
\hline Study & $\begin{array}{c}\text { Number of cases } \\
\text { (hydrops/ CVR >1.6) }\end{array}$ & $\begin{array}{l}\text { GA at steroids (wks) } \\
\text { Median (range) }\end{array}$ & $\begin{array}{l}\text { CVR at steroids }\left(\mathrm{cm}^{2}\right) \\
\text { Median (range) }\end{array}$ & $\begin{array}{c}\text { Hydrops resolution } \\
\mathrm{n}(\%)\end{array}$ & $\begin{array}{c}\text { Overall survival rate } \\
\mathrm{n}(\%)\end{array}$ \\
\hline Tsao et al, 2002 (2) & 3 & $22(21-27)$ & - & $3(100 \%)$ & $3(100 \%)$ \\
\hline Peranteau et al, 2007 (39) & 5 & - & $\geq 1.6$ & $4 / 5(80 \%)$ & $11(100 \%)$ \\
\hline Curran et al, 2009 (40) & 13 & $24.55 \pm 1.53$ & $2.72 \pm 0.92$ & $7 / 9(77.8 \%)$ & $11(84.6 \%)$ \\
\hline Loh et al, 2011 )36) & 13 & $23(20-26)$ & $2.68 \pm 0.29$ & $10 / 13(77 \%)$ & $10(76 \%)$ \\
\hline Yamashita et al, 2014 (42) & 3 & $22(21-23)$ & $2.43 \pm 1.19$ & $2 / 2(100 \%)$ & $3(100 \%)$ \\
\hline
\end{tabular}

GA - gestational age; wks - gestational weeks; CVR - CPAM volume ratio

prenatal steroids in microcystic CPAM in three fetuses who presented with hydrops, with $100 \%$ survival rate. Peranteau et al (39) reported $100 \%(11 / 11)$ survival after administrating betamethasone, $80 \%(4 / 5)$ hydrops resolution. Curran et al (40) reported following results after betamethasone administration: decrease of CVR in $61.5 \%(8 / 13)$ and $77.8 \%$ (7/9) hydrops resolution rate, $100 \%$ fetuses survived to delivery and $84.6 \%$ survived to neonatal discharge. Two fetuses whose hydrops did not resolve did not survive to discharge. Loh et al (36) reported $77 \%$ (10/13) survival rate after administration of betamethasone while Derderian et al (41) published $63 \%$ (5/8) survival after repeated administration of betamethasone. Yamashita et al (42), published $100 \%(3 / 3)$ survival after single dose of betamethasone and 100 $\%$ hydrops resolving. Peranteau et al (43) monitored the effect of betamethasone in single dose and multiple dosing, with result of $86 \%$ survival in the multiple administration of betamethasone vs $93 \%$ of a single dose of betamethasone. Review of the published data on standard single dose betamethasone treatment of CPAM can be seen in Table 1. For comparison, Adzick (44) describes 24 cases of open surgery with 11 cases of fetal death. Regarding above data, there is strong evidence that prenatal steroid administration represents first choice treatment in fetuses with highrisk CPAM. It is still unclear if it is also beneficial for patients with non-hydropic microcystic/other CPAM lesions. It is mainly due to a lack of experience with the effect of prenatal steroids on non-hydropic CPAM fetuses and concerns about different response and effects on the child's future health. On the other hand, even though CPAM is suggested to be disease with good prognosis in case it does not perform with hydrops $(11,21,45)$, there is still significant morbidity during postnatal period described in direct association with $\operatorname{CPAM}(12,22,24)$. This is in accordance with the mentioned finding of systematic review suggesting that threshold of CVR for perinatal outcomes is different and lower than threshold for fetal hydrops (38). Moreover, Gallardo (11) states the prenatal resolution of the lesion should not be accepted as evidence of a true disappearance of the pathology as $45 \%$ of the lesions considered absent in the Nicolaides series (10) were subsequently subjected to resection due to the existence of persistent lesions on CT.

We described 4 cases of prenatally diagnosed CPAM at 20-26 gw, 2 microcystic and two mixed forms. Even though none of the cases performed hydrops, one case presented with mediastinal shift and CVR close to the risk value. In the other cases, CPAM lesions continued to grow even after $28 \mathrm{gw}$. Betamethasone in standard regimen of 2 doses, $12 \mathrm{mg}$ intramuscularly, 24 hours apart was administered in all of the cases. All fetuses responded to the treatment with either stagnation or regression of the size of the CPAM mass. We did not record complete resolution of the mass in any of the patients nor did we observe other complications of the pregnancy that could be related to the steroid treatment. All patients delivered at $37 \mathrm{gw}$ or later after spontaneous onset of labor or premature rupture of membranes without complications, 2 delivered vaginally, 2 by caesarean section due to previous caesarean section and placenta previa. All children survived until discharge and all of them had to undergo thoracoscopic pulmonary resection of the remaining lesion during first year of life. The fact that postnatally diagnosed persistent pulmonary mass diagnosed by CT was larger than that diagnosed prenatally by ultrasound may be explained by the results of Nicolaides series (10).

We currently continue to follow up the presented children and collect data regarding their postnatal development and health state. Even though our case series does not prove the benefit of antenatal administration of betamethasone in non-hydropic fetuses with prenatally diagnosed CPAM, we believe it supports the "safety" of the betamethasone administration in these cases at least during the perinatal period.

The current recommendations to treat only high-risk fetuses with CPAM come from the concerns about the possible effects of the antenatal corticosteroids to the child. However, there are already several longer-term follow-up studies such as the extended original Liggins and Howie randomized cohort (46) that were followed up at age 30 years. Even though the betamethasone in this study was administered in order to manage patients with imminent preterm birth, data show that exposure to $12 \mathrm{mg}$ betamethasone, two doses $24 \mathrm{~h}$ apart or double this dose did not alter growth parameters or cardio-metabolic risk, the only difference being a higher insulin level after glucose tolerance test, suggesting possible mild insulin resistance. Psychological testing showed no difference in cognitive functioning, working memory and attention, psychiatric morbidity, handedness, or quality of life (47). Considering our data, uncomplicated course of the pregnancy after betamethasone administration, calculating the risk and benefit together with its non-invasive and simple use, we think that all patients with prenatally diagnosed CPAM could be offered this treatment as soon as the diagnosis is set, particularly patients requesting active management. We believe it is more beneficial to start the steroid therapy before the hydrops occurs, as this state may already be associated with brain perfusion failure and ischemic changes (24). Moreover, the treatment might help to avoid other complications such as pulmonary compression of any grade, 
risk of premature labor or at the very least might help to block the lesion growth at the very beginning and ensure its extent as small as possible. This is particularly important for the postnatal period and extent of the surgery, which is needed in most cases. Early betamethasone administration may reduce the need of open postnatal surgery in favor of thoracoscopic approach and may positively influence overall morbidity in children with history of CPAM. Up to now there is no study that would analyze the effect of betamethasone on non-hydropic CPAM fetus in relation to overall outcome and postnatal morbidity. This remains challenge for the future studies that are needed for decision making in the prenatal management of CPAM.

\section{References}

1. Khalek N, Johnson MP. Management of prenatally diagnosed lung lesions. Semin Pediatr Surg 2013; 24-29.

2. Tsao K, Hawgood S, Vu L et al. Resolution of hydrops fetalis in congenital cystic adenomatoid malformation after prenatal steroid therapy. $\mathrm{J}$ Pediatr Surg 2003; 38 (3): 508-510.

3. Iavazzo C, Eleftheriades M, Bacanu AM, Hassiakos D, Botsis D. Congenital cystic adenomatoid malformation: is there a need for pregnancy termination? Case Rep Med 2012; 2012: 397508.

4. Sfakianaki AK, Copel JA. Congenital cystic lesions of the lung: congenital cystic adenomatoid malformation and bronchopulmonary sequestration. Rev Obstet Gynecol 2012; 5 (2): 85.

5. Adzick NS, Kitano Y. Fetal surgery for lung lesions, congenital diaphragmatic hernia, and sacrococcygeal teratoma. Semin Pediatr Surg 2003; 12 (3): 154-167.

6. Crombleholme TM, Coleman B, Hedrick $\mathrm{H}$ et al. Cystic adenomatoid malformation volume ratio predicts outcome in prenatally diagnosed cystic adenomatoid malformation of the lung. J Pediatr Surg 2002; 37: 331-338.

7. Di Prima FAF, Bellia A, Inclimona G, Grasso F, Teresa M, Cassaro MN. Antenatally diagnosed congenital cystic adenomatoid malformations (CCAM): Research Review. J Prenat Med 2012; 6 (2): 22.

8. Silva E, Vale J, Reis A. Late onset congenital cystic adenomatous malformation associated with intra-lobar pulmonary sequestration. Rev Port Pneumol 2015; 21 (5): 282-284.

9. Ierullo AM, Ganapathy R, Crowley S, Craxford L, Bhide A, Thilaganathan B. Neonatal outcome of antenatally diagnosed congenital cystic adenomatoid malformations. Ultrasound Obstet Gynecol 2005; 26 (2): $150-153$.

10. Davenport M, Warne SW, Cacciaguerra S, Patel S, Greenough A, Nicolaides K. Current outcome of antenatally diagnosed cystic lung disease. J Pediatr Surg 2004; 4: 549-556.

11. Gallardo MA, Álvarez de la Rosa M, De Luis JF, Mendosa L, Padilla AI, Troyano J. Antenatal ultrasound diagnosis and neonatal results of the congenital cystic adenomatoid malformation of the lung. Rev Chil Pediatr 2018; 89 (2): 224-230.

12. Hancock BJ, Di Lorenzo M, Youssef S, Yazbeck S, Marcotte JE, Collin PP. Childhood primary pulmonary neoplasms. J Pediatr Surg 1993; 28: $1133-1136$

13. Laberge JM, Puligandla P, Flageole H. Asymptomatic congenital lung malformations. Semin Pediatr Surg 2005; 14: 16-33.
14. Stocker JT, Madewell JE, Drake RM. Congenital cystic adenomatoid malformation of the lung classification and morphologic spectrum. Hum Pathol 1977; 8: 155-171.

15. Stocker JT. Congenital pulmonary airway malformation - a new name for and an expanded classification of congenital cystic adenomatoid malformation of the lung. Symposium 24: non-neoplastic lung disease. Histopathology 2002; 41 (Suppl. 2): 424-430.

16. Dos Reis AR, Ribeiro FB, Schultz R. Congenital cystic adenomatoid malformation type I. Autops Case Rep 2015; 5 (3): 21-26.

17. Durell J, Lakhoo K. Congenital cystic lesions of the lung. Early Hum Dev 2014; 90(12): 935-939.

18. Annunziata F, Bush A, Borgia F et al. Congenital Lung Malformations: Unresolved Issues and Unanswered Questions. Front Pediatr Published online: 13 June 2019. https://doi.org/10.3389/fped.2019.00239.

19. Adzick NS, Harrison MR, Glick PL et al. Fetal cystic adenomatoid malformation: prenatal diagnosis and natural history. J Pediatr Surg 1985; 20 (5): 483-488.

20. Adzick NS, Flake AW, Crombleholme TM. Management of congenital lung lesions. Semin Pediatr Surg 2003; 12 (1): 10-16.

21. Witlox RS, Lopriore E, Oepkes D. Prenatal interventions for fetal lung lesions. Prenat Diagn 2011; 31 (7): 628-636.

22. Williams HJ, Johnson KJ. Imaging of congenital cystic lung lesions. Paediatr Respir Rev 2002; 3: 120-127.

23. Alamo L, Gudinchet $F$, Reinberg $O$ et al. Prenatal diagnosis of congenital lung malformations. Pediatr Radiol 2012; 42: 273-283.

24. Larroche JC, Aubry MC, Narcy F. Intrauterine brain damage in nonimmune hydrops fetalis. Biol Neonate 1992; 61 (5): 273-280.

25. Baird R, Puligandla PS, Laberge JM. Congenital lung malformations: informing best practice. Semin Pediatr Surg 2014; 23: 270-277.

26. Bush A. Rare lung diseases: congenital malformations. Indian J Pediatric 2015; 82: 833-840.

27. Quintero RA, Kontopoulos E, Reiter J, Pedreira WL, Colin AA. Fetal bronchoscopy: its successful use in a case of extralobar pulmonary sequestration. J Matern Fetal Neonatal Med 2012; 25: 2354-2358.

28. Cruz-Martinez R, Méndez A, Perez-Garcilita $O$ et al. Fetal bronchoscopy as a useful procedure in a case with prenatal diagnosis of congenital microcystic adenomatoid malformation. Fetal Diagn Ther 2015; 37: $75-80$.

29. Knox EM, Kilby MD, Martin WL, Khan KS. In-utero pulmonary drainage in the management of primary hydrothorax and congenital cystic lung lesion: a systematic review. Ultrasound Obstet Gynecol 2006; 28: 726-734.

30. Sauvat F, Michel JL, Benachi A, Emond S, Revillon Y. Management of asymptomatic neonatal cystic adenomatoid malformations. J Pediatr Surg 2003; 38: 548-552.

31. Cavoretto P, Molina F, Poggi S, Davenport M, Nicolaides KH. Prenatal diagnosis and outcome of echogenic fetal lung lesions. Ultrasound Obstet Gynecol 2008; 32: 769-783.

32. Miller JA, Corteville JE, Langer JC. Congenital cystic adenomatoid malformation in the fetus: natural history and predictors of outcome. J Pediatr Surg 1996; 31: 805-808.

33. Adzick NS, Harrison MR, Flake AW, Howell LJ, Golbus MS, Filly RA. Fetal surgery for cystic adenomatoid malformation of the lung. J Pediatr Surg 1993; 28: 806-812. 
657-662

34. Jain L, Eaton DC. Physiology of fetal lung fluid clearance and the effect of labor. Semin Perinatol 2006; 30: 34-43.

35. Morris LM, Lim FY, Livingston JC, Polzin WJ, Crombleholme TM. High-risk fetal congenital pulmonary airway malformations have a variable response to steroids. J Pediatr Surg 2009; 44: 60-65.

36. Loh KC, Jelin E, Hirose S, Feldstein V, Goldstein R, Lee H. Microcystic congenital pulmonary airway malformation with hydrops fetalis: steroids vs open fetal resection. J Pediatr Surg 2012; 47: 36-39.

37. David M, Lamas-Pinheiro R, Henriques-Coelho T. Prenatal and Postnatal Management of Congenital Pulmonary Airway Malformation. Neonatology 2016; 110: 101-115.

38. Kane SC, Ancona E, Reidy KL, Palma-Dias R. The Utility of the Congenital Pulmonary Airway Malformation-Volume Ratio in the Assessment of Fetal Echogenic Lung Lesions: A Systematic Review. Fetal Diagn Ther 2020; 47 (3): 171.

39. Perenteau WH, Wilson RD, Liechty KW et al. Effect of maternal betamethasone administration on prenatal congenital cystic adenomatoid malformation growth and fetal survival. Fetal Diagn Ther 2007; 22 (5): 365-371.

40. Curran PF, Jelin EB, Rand L et al. Prenatal steroids for microcystic congenital cystic adenomatoid malformations. J Pediatr Surg 2010; 45: $145-150$.
41. Derderian SC, Coleman AM, Jeanty C et al. Favorable outcomes in high-risk congenital pulmonary airway malformations treated with multiple courses of maternal betamethasone. J Pediatr Surg 2015; 5 (4): 515-518.

42. Yamashita A, Hidaka N, Yamamoto R et al. In utero resolution of microcystic congenital cystic adenomatoid malformation after prenatal betamethasone therapy: A report of three cases and a literature review. J Clin Ultrasound 2015; 43 (7): 451-457.

43. Peranteau WH, Boelig MM, Khalek $\mathbf{N}$ et al. Effect of single and multiple courses of maternal betamethasone on prenatal congenital lung lesion growth and fetal survival. J Pediatr Surg 2016; 51 (1): 28-32.

44. Adzick NS. Management of fetal lung lesions. Clin Perinatol 2009; 36: 363-376.

45. Bunduki V, Ruano R, da Silva MM et al. Prognostic factors associated with congenital cystic adenomatoid malformation of the lung. Prenat Diagn 2000; 20 (6): 459-464.

46. Dalziel SR, Walker NK, Parag V et al. Cardiovascular risk factors after antenatal exposure to betamethasone: 30 -year follow-up of a randomised controlled trial. Lancet 2005; 365 (9474): 1856-1862.

47. Dalziel SR, Lim VK, Lambert A et al. Antenatal exposure to betamethasone: psychological functioning and health related quality of life 31 years after inclusion in randomised controlled trial. BMJ 2005; 331 (7518): 665. 